\title{
Measurement of pulse amplitude and phase distortion in a semiconductor optical amplifier: from pulse compression to breakup
}

Romstad, Francis Pascal; Borri, Paola; Langbein, Wolfgang Werner; Mørk, Jesper; Hvam, Jørn Märcher

Published in:

I E E E Photonics Technology Letters

Link to article, DOI:

$10.1109 / 68.896345$

Publication date:

2000

Document Version

Publisher's PDF, also known as Version of record

Link back to DTU Orbit

Citation (APA):

Romstad, F. P., Borri, P., Langbein, W. W., Mørk, J., \& Hvam, J. M. (2000). Measurement of pulse amplitude and phase distortion in a semiconductor optical amplifier: from pulse compression to breakup. I E E E Photonics Technology Letters, 12(12), 1674-1676. https://doi.org/10.1109/68.896345

\section{General rights}

Copyright and moral rights for the publications made accessible in the public portal are retained by the authors and/or other copyright owners and it is a condition of accessing publications that users recognise and abide by the legal requirements associated with these rights.

- Users may download and print one copy of any publication from the public portal for the purpose of private study or research.

- You may not further distribute the material or use it for any profit-making activity or commercial gain

- You may freely distribute the URL identifying the publication in the public portal 


\title{
Measurement of Pulse Amplitude and Phase Distortion in a Semiconductor Optical Amplifier: from Pulse Compression to Breakup
}

\author{
F. Romstad, P. Borri, W. Langbein, J. Mørk, and J. M. Hvam
}

\begin{abstract}
We have performed extensive measurements of the propagation of ultrashort pulses in a semiconductor bulk amplifier using an ultrasensitive cross frequency-resolved optical gating technique. Pulses of 175-fs duration with energies from below $1 \mathrm{fJ}$ to above $100 \mathrm{pJ}$ are measured both in amplitude and phase after propagation through the device. While only moderate reshaping effects occur at pulse energies of below $1 \mathrm{pJ}$, strong amplitude distortion together with nonlinear chirp is found for input energies of 5-100 pJ. This leads to a pulse narrowing by more than a factor of two when the amplifier is biased for material transparency or absorption and to a pronounced pulse breakup in the gain regime.
\end{abstract}

Index Terms-Distortion, optical communication, optical propagation in nonlinera media, optical pulse compression, optical pulse measurements, propagation, semiconductor device measurements, semiconductor optical amplifiers.

\section{INTRODUCTION}

$\mathbf{S}$ EMICONDUCTOR optical amplifiers (SOAs) are attracting increasing interest in the field of telecommunications due to their small size, high gain, and electrooptical properties. Recently, 640-Gbit/s single-channel transmission has been demonstrated [1]. Thus, the study of the transmission characteristics of subpicosecond pulses in SOAs is becoming of practical relevance. Propagation and distortion of ultrashort pulses in SOAs was experimentally investigated in the past, however, only using characterization techniques such as autocorrelation, cross-correlation and spectral-intensity measurements [2], [3]. A direct phase-sensitive diagnostic of the pulse was missing. The knowledge of the pulse chirp acquired after transmission through an SOA is crucial for the application of these components in optical communications at high bit-rates, as well as for the understanding of the physical processes involved in the pulse distortion, as discussed in several theoretical works [3], [5].

The simultaneous characterization of amplitude and phase of an optical pulse is a complicated task. One of the first attempts of amplitude and phase retrieval of subpicosecond pulses was based on a combination of first-order interferometric correlation and time-resolved intensity measurements [4]. More recently,

Manuscript received July 5, 2000; revised September 11, 2000. The work was supported by the Danish Technical Research Council under the framework of SCOOP (Semiconductor Components for Optical Signal Processing).

F. Romstad, J. Mørk, and J. M. Hvam are with the Research Center COM, Technical University of Denmark, DK-2800 Lyngby, Denmark.

P. Borri and W. Langbein are with the Lehrstuhl fur Experimentelle Physik

EIIb, Universitat Dortmund, 44227 Dortmund, Germany.

Publisher Item Identifier S 1041-1135(00)10480-X. the full characterization of ultrashort pulses was made possible by the introduction of frequency-resolved optical gating (FROG) techniques together with robust generalized projection algorithms to retrieve the complex electrical field [6]. The FROG technique has been adopted in many fields of ultrashort pulse characterization, such as pulses of few optical cycles [7], regenerative pulse amplification [8], and pulse propagation in nonlinear dispersive media [9]. The introduction of second harmonic generation FROG (SHG-FROG) has considerably increased the sensitivity compared to FROG techniques based on $\chi^{3}$ processes [6]. Characterization of pulses with energies of the order of $20 \mathrm{pJ}$ has been reported recently [10]. However, for telecommunication purposes, pulse characterization at lower energy $(<1 \mathrm{pJ})$ is important.

In this letter, we apply an altered FROG technique to investigate the propagation of 175 -fs pulses in an InGaAsP-InP bulk SOA operating at a $1.53 \mu \mathrm{m}$ wavelength. The technique, called cross-frequency-resolved optical gating (XFROG) [11] is based on the principle of crosscorrelation. The pulse to be characterized is correlated with a strong reference pulse, increasing the sensitivity of the technique compared to traditional FROG. In addition, the algorithm for pulse retrieval is converging faster, using a known reference pulse, and has no time ambiguity compared to the FROG solution [11]. We present measurements on the pulse amplitude and phase distortion over a wide range of pulse energies, ranging from below $1 \mathrm{fJ}$ to above $100 \mathrm{pJ}$. Pulse propagation is investigated in the three regimes of operation of the SOA: gain, transparency and absorption. The transparency current was determined using pump-probe [12]. Pulses of $175-$ fs duration at $1.53 \mu \mathrm{m}$ wavelength from a $300-\mathrm{kHz}$ repetition-rate optical parametric amplifier (OPA) are coupled into and out of a $250-\mu \mathrm{m}$ long bulk SOA, using high numerical aperture lenses. When operating in the gain regime at $120 \mathrm{~mA}$, $1.53-\mu \mathrm{m}$ wavelength corresponds to the maximum of the spectral gain of the device giving $14-\mathrm{dB}$ modal gain. After propagation through the device the pulses are focused into a $0.4-\mathrm{mm}$ BBO crystal and cross-correlated with reference pulses of $\sim 100$ $\mathrm{nJ}$ energies at $800-\mathrm{nm}$ wavelength produced by a regenerative amplifier (Coherent RegA 9000). The spectrum of the up-converted signal intensity is recorded as a function of the delay between reference and signal pulses, using a spectrometer and a charge coupled device (CCD) camera with $0.3-\mathrm{nm}$ resolution. A two-dimensional XFROG trace consisting of a $256 \times 256$ array, with the wavelength along one axis and the delay time along the other, is then constructed. To retrieve the pulse from this trace, the electrical field of the reference pulse must be known, which 
we obtain with traditional FROG. The strong pulse energy of the reference pulse ensures a high signal-to-noise ratio and, therefore, an easy retrieval for the FROG-algorithm evidenced in the low FROG-errors 0.003 [11]. Both for the FROG and the XFROG, an iterative Fourier-transform algorithm with generalized projection is applied [11]. To investigate the distortion induced by propagation through the SOA the pulse at the input of the device was characterized. Fig. 1(a) shows the temporal amplitude and instantaneous frequency of the input pulse, taken with 10 fs step size. The full-width at half-maximum (FWHM) of the pulse intensity is $175 \mathrm{fs}$. The instantaneous frequency $(\omega)$ is defined from the phase $(\phi)$ using

$$
\omega=-\frac{1}{2 \pi} \frac{d \phi}{d t}
$$

The input pulse is nearly transform-limited, since the instantaneous frequency is close to constant. In Fig. 1(b), the retrieved spectrum is shown. The measured time-bandwidth product is 0.44 .

The pulse at the output of the SOA under bias current, corresponding to 14-dB small-signal gain, is shown in Fig. 2(a), for input pulse energies ranging from $0.2 \mathrm{fJ}$ to $27 \mathrm{pJ}$. Note that the pulse with energy of $0.2 \mathrm{fJ}$, corresponding to $50 \mathrm{fJ}$ at the output, is well characterized, with a high signal-to-noise ratio (FROG error 0.004), and is still almost Fourier-limited. For increasing pulse energies nonlinear gain saturation occurs, as shown in Fig. 2(b). At input energies of $>0.1 \mathrm{pJ}$ two-photon absorption (TPA) leads to negative gain values. At $1 \mathrm{pJ}$ input energy, the gain has decreased from 14 to $-7 \mathrm{~dB}$. Here, a weak distortion of the temporal pulse shape is evidenced as a time broadening together with a weak modulation of the instantaneous frequency, as shown in Fig. 2(a).

This broadening turns into a pulse break up at $5 \mathrm{pJ}$ input energy (not shown). At $27 \mathrm{pJ}$ input energy, the break up is completely evolved with a distance of $\sim 650 \mathrm{fs}$ between the two maxima [see Fig. 2(a)] which persists for further increase of the pulse energy up to $100 \mathrm{pJ}$. The instantaneous frequency is highly nonlinear for input energies $>5 \mathrm{pJ}$, indicating the occurrence of self-phase modulation consistent with a Kerr nonlinearity associated with the TPA.

At transparency bias current the pulse distortion is very different, as shown in Fig. 3.

Instead of a break-up in time, a pulse narrowing is measured at high input energies. The output pulse at low energy (1 fJ) resembles the input pulse, showing that the SOA is working in the linear regime. For an input energy of $1 \mathrm{pJ}$, a weak time narrowing is observed, that evolves up to a narrowing of more than a factor of two at 106-pJ input energy. The signal arriving before the narrowed pulse for the 106-pJ input pulse is recognized as unguided light. The additional information on the instantaneous frequency shows that the narrowed pulse is not transform-limited and a negative chirp is observed. In absorption, corresponding to $-6 \mathrm{~dB}$ small signal gain, we observe a similar phenomenon as for transparency, namely a pulse narrowing for increasing pulse energies. A detailed modeling of the subpicosecond pulse propagation through an SOA, especially at high input energy where highly nonlinear effects occur, is a complicated task. Both phenomenological

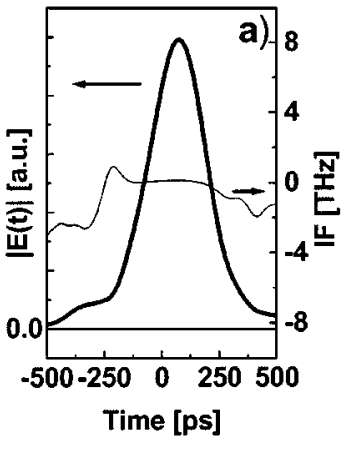

(a)

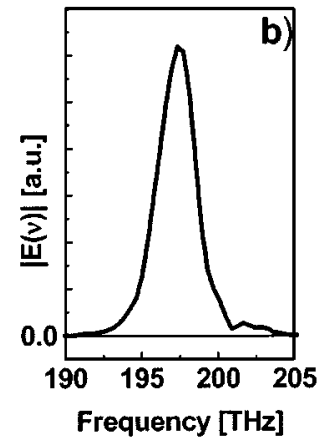

(b)
Fig. 1. (a) The electrical field of the excitation pulse in the time domain together with the instantaneous frequency (IF). (b) The electrical field in the frequency domain.

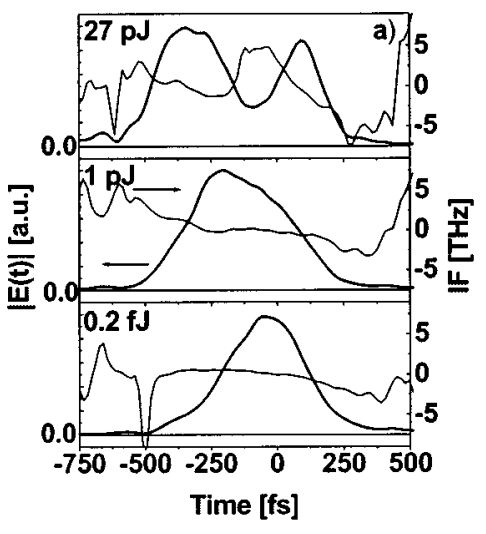

(a)

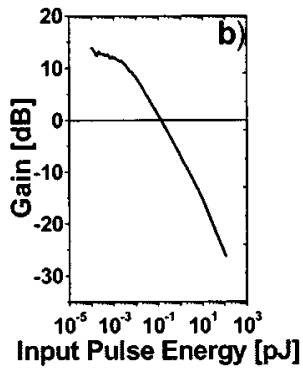

(b)
Fig. 2. (a) Temporal pulse field after transmission through the device, biased for 14-dB small-signal gain, for different input pulse energies. (b) Internal device gain as function of input pulse energy.

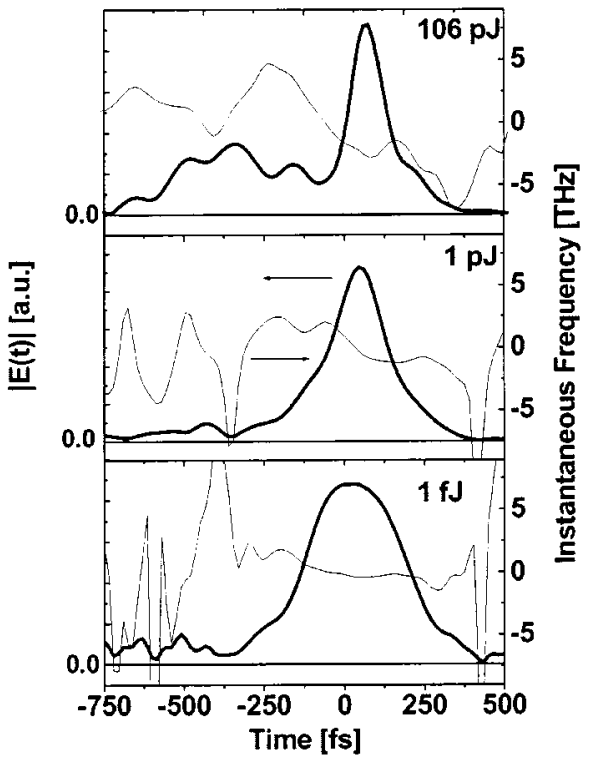

Fig. 3. Output pulse in the time-domain after propagation through the device, biased at transparency, for various input energies.

[3], [13] and microscopic many-body [14] calculations have been proposed to solve this problem. Pulse propagation at the maximum spectral gain that evolves into a strong break-up after few hundred microns of propagation distance was predicted 
by Hughes [14], similar to our results. However, only small pulse reshaping was predicted at transparency and absorption, different from our measurements. In general, a complicated interplay between several microscopic processes, such as nonlinear gain saturation, TPA, gain and group-velocity dispersion, nonadiabatic following, propagation effects, is responsible for the observed distortion and the detailed modeling of these processes is beyond the scope of this letter. However, the experimental determination of the pulse chirp shown in this work should bring additional information in the understanding of this complicated scenario.

In conclusion, we have presented the complete diagnostic, in amplitude and phase, of the propagation of 175-fs pulses through a bulk semiconductor optical amplifier, over a wide range of pulse energies from below $1 \mathrm{fJ}$ to above $100 \mathrm{pJ}$. At pulse energies of 5-100 pJ strong distortions, going from pulse narrowing by more than a factor of two in the transparency and absorption case to pulse breakup in the gain case, have been measured. From our results we conclude that the use of SOAs for high speed/high power applications can undergo severe limitations due to pulse distortion for pulse durations of a few hundred femtoseconds and energies above a few picajoules. Alternatively, possible applications of SOA's in pulse shaping techniques could be of interest.

\section{ACKNOWLEDGMENT}

The authors gratefully acknowledge S. Linden for providing the X-FROG algorithm.

\section{REFERENCES}

[1] M. Nakazawa, "Toward terabit/s single-channel transmission," in Proc. OFC99, 1999, FI1-1, p. 132.
[2] Y.-H. Kao, I. V. Goltser, M. Jiang, M. N. Islam, and G. Raybon, "Gain dispersion induced subpicosecond pulse breakup in a fiber and semiconductor laser amplifier combined system," Appl. Phys. Lett., vol. 69, no. 27, pp. 4221-3, 1996.

[3] M. Y. Hong, Y. H. Chang, A. Dienes, J. P. Heritage, and P. J. Delfyett, "Subpicosecond pulse amplification in semiconductor laser amplifiers: Theory and experiment," IEEE J. Quantum Electron., vol. 30, no. 4, pp. 1122-1131, 1994.

[4] J.-Y. Bigot, M.-A. Mycek, S. Weiss, R. G. Ulbrich, and D. S. Chemla, "Instantaneous frequency dynamics of coherent wave mixing in semiconductor Quantum Wells," Phys. Rev. Lett., vol. 70, pp. 3307-3310, 1993.

[5] S. Hughes, A. Knorr, and S. W. Koch, "Femtosecond pulse breakup in a semiconductor amplifier," Opt. Lett., vol. 21, no. 14, pp. 1052-1054, 1996.

[6] K. W. DeLong, R. Trebino, J. Hunter, and W. E. White, "Frequencyresolved optical gating with the use of second-harmonic generation," $J$. Opt. Soc. Amer. B, vol. 11, no. 11, pp. 2206-15, 1994.

[7] Z. Cheng, A. Furbach, S. Sartania, M. Lenzner, C. Spielmann, and F. Krausz, "Amplitude and chirp characterization of high-power laser pulses in the 5-fs regime," Opt. Lett., vol. 24, no. 4, pp. 247-9, 1999.

[8] B. Kohler, V. V. Yakovlev, K. R. Wilson, J. Squier, K. W. DeLong, and R. Trebino, "Phase and intensity characterization of femtosecond pulses from a chirped-pulse amplifier by frequency-resolved optical gating," Opt. Lett., vol. 20, no. 5, pp. 483-5, 1995.

[9] S. A. Diddams, H. K. Eaton, A. A. Zozulya, and T. S. Clement, "Amplitude and phase measurements of femtosecond pulses splitting in nonlinear dispersive media," Opt. Lett., vol. 23, no. 5, pp. 379-381, 1998.

[10] F. G. Omenetto, J. W. Nicholson, and A. J. Taylor, "Second-harmonic generation-frequency-resolved optical gating analysis of low-intensity shaped femtosecond pulses at $1.55 \mu \mathrm{m}$," Opt. Lett., vol. 24, no. 23, pp. 1780-2, 1999.

[11] S. Linden, H. Giessen, and J. Kuhl, "XFROG-A new method for amplitude and phase characterization of weak ultrashort pulses," Phys. Stat. Sol. B, vol. 206, pp. 119-24, 1998.

[12] P. Borri, W. Langbein, J. Mørk, and J. M. Hvam, "Heterodyne pump-probe and four-wave mixing in semiconductor optical amplifiers using balanced lock-in detection," Opt. Commun., vol. 169, pp. 317-324, 1999

[13] J. Mork, J. Mark, and C. P. Seltzer, "Carrier heating in InGaAsP laser amplifiers due to two-photon absorption," Appl. Phys. Lett., vol. 64, no. 17, pp. 2206-2208, 1994.

[14] S. Hughes, "Carrier-carrier interaction and ultrashort pulse propagation in a highly excited semiconductor laser amplifier beyond the rate equation limit," Phys. Rev. A, vol. 58, no. 3, pp. 2567-2576, 1996. 